\title{
BMJ
}

\section{Efficacy of self monitoring of blood glucose in patients with newly diagnosed type 2 diabetes (ESMON study): randomised controlled trial}

\author{
Maurice J O'Kane, consultant, ${ }^{1}$ Brendan Bunting, professor, ${ }^{2}$ Margaret Copeland, trial manager, ${ }^{3}$ \\ Vivien E Coates, professor, ${ }^{3}$ on behalf of the ESMON study group
}

\begin{abstract}
${ }^{1}$ Department of Clinical Chemistry, Altnagelvin Hospital, Western Health and Social Care Trust, Londonderry, Northern Ireland

${ }^{2}$ Psychology Research Institute, University of Ulster, Londonderry, Northern Ireland

${ }^{3}$ Institute of Nursing Research, University of Ulster, Coleraine, Northern Ireland
\end{abstract}

Correspondence to: M J O'Kane Maurice.OKane@westerntrust. hscni.net

doi:10.1136/bmj.39534.571644.BE

\section{ABSTRACT}

Objectives To assess the effect of self monitoring of blood glucose concentrations on glycaemic control and psychological indices in patients with newly diagnosed type 2 diabetes mellitus.

Design Prospective randomised controlled trial of self monitoring versus no monitoring (control).

Setting Hospital diabetes clinics.

Participants 184 (111 men) people aged <70 with newly diagnosed type 2 diabetes referred to the participating diabetes clinics. Major exclusion criteria were secondary diabetes, insulin treatment, previous self monitoring of blood glucose.

Interventions Participants were randomised to self monitoring or no monitoring (control) groups for one year with follow-up at three monthly intervals. Both groups underwent an identical structured core education programme. The self monitoring group received additional education on monitoring.

Main outcome measures Between group differences in $\mathrm{HbA}_{1 \mathrm{c}}$, psychological indices, use of oral hypoglycaemic drugs, body mass index (BMI), and reported

hypoglycaemia rates.

Results 96 patients ( 55 men) were randomised to monitoring and 88 ( 56 men) to control. There were no baseline differences in mean (SD) age (57.7 (11.0) in monitoring group $v 60.9$ (11.5) in control group) or $\mathrm{HbA}_{1 \mathrm{c}}$ (8.8 (2.1)\% $v 8.6$ (2.3)\%, respectively). Those in the monitoring group had a higher baseline BMI (34 (7) $v 32$ (6.2)). There were no significant differences between groups at any time point (12 months values given) in $\mathrm{HbA}_{1 \mathrm{c}}(6.9$ (0.8)\% v 6.9 (1.2)\%, $\mathrm{P}=0.69 ; 95 \%$ confidence interval for difference $-0.25 \%$ to $0.38 \%$ ), BMI (33.1 (6.4) $v$ 31.8 (6.0); adjusted for baseline $\mathrm{BMI}, \mathrm{P}=0.32$ ), use of oral hypoglycaemic drugs, or reported incidence of hypoglycaemia. Monitoring was associated with a $6 \%$ higher score on the depression subscale of the well-being questionnaire $(P=0.01)$.

Conclusions In patients with newly diagnosed type 2 diabetes self monitoring of blood glucose concentration has no effect on glycaemic control but is associated with higher scores on a depression subscale.

Trial registration ISRCTN 49814766.

\section{INTRODUCTION}

Although self monitoring of blood glucose concentrations is widely advocated by healthcare professionals for patients with type 2 diabetes mellitus who do not require insulin, there is conflicting evidence as to its value. ${ }^{1}$ Self monitoring might contribute to management in two ways. Firstly, it might improve glycaemic control by reinforcing beneficial self management behaviours and compliance with medication. Secondly, the process of monitoring and the immediate feedback it provides on glycaemic control might affect patients' experience and determine attitudes to their diabetes and satisfaction with treatment.

We investigated the effect of self monitoring on glycaemic control and attitudes and satisfaction with treatment in patients with newly diagnosed type 2 diabetes.

\section{METHODS}

The ESMON study was a randomised controlled trial of self monitoring of blood glucose concentration (intervention) versus no monitoring (control). Patients aged $<70$ with newly diagnosed type 2 diabetes were recruited from the outpatient diabetes services at Altnagelvin, Belfast City, Causeway, and the Ulster Hospitals (Northern Ireland) between 2002 and 2005. The decision to refer individual patients reflected the normal referral practice of the primary care doctor. Exclusion criteria included secondary diabetes, use of insulin, previous use of self monitoring of blood glucose, major illness within the previous six months, chronic kidney disease, chronic liver disease, and alcohol misuse.

\section{Outcomes}

Our pre-designated primary end points were differences between groups in $\mathrm{HbA}_{1 \mathrm{c}}$, psychological indices, and incidence of hypoglycaemia. Our secondary end points were differences between groups in body mass index and use of oral hypoglycaemic drugs.

\section{Randomisation}

Participants were recruited from among those patients with newly diagnosed type 2 diabetes referred to the hospital diabetes clinics. 


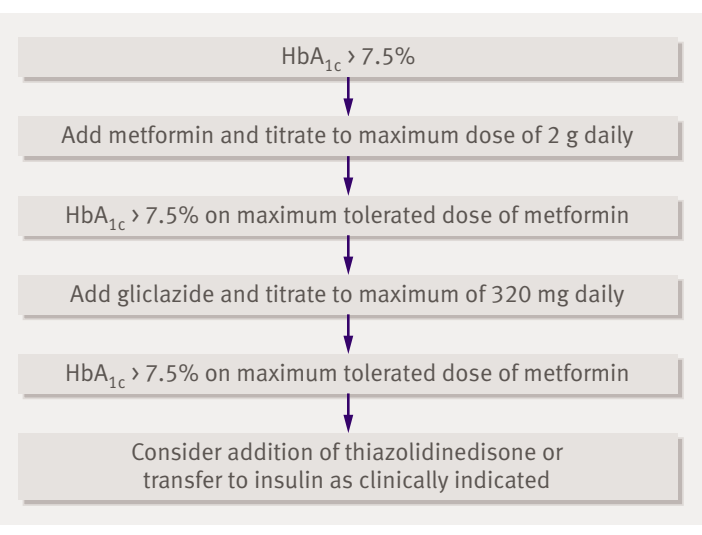

Fig 1| Treatment algorithm for oral hypoglycaemic agents

After an initial assessment visit, eligible patients were randomised into intervention (self monitoring of blood glucose) or control (no monitoring) groups with a randomly generated allocation code in consecutively numbered sealed envelopes. The study diabetes nurse at each hospital site performed the treatment allocation.

Patients in the self monitoring group were all provided with a single glucose monitor (Lifescan OneTouch Ultra; Johnson and Johnson, Milpitas, CA) and instructed in its use. They were asked to monitor four fasting and four postprandial capillary blood glucose measurements each week. They were advised on appropriate responses to high or low readings. Such advice included the need for dietary review or the suggestion of exercise (such as walking) in response to high readings. At each clinic visit, concordance with the self monitoring regimen was verified by downloading meter readings.

Patients in the no monitoring group (control) were asked not to acquire a meter or perform monitoring for the duration of the study.

Patients in both groups underwent an identical structured education programme involving diabetes

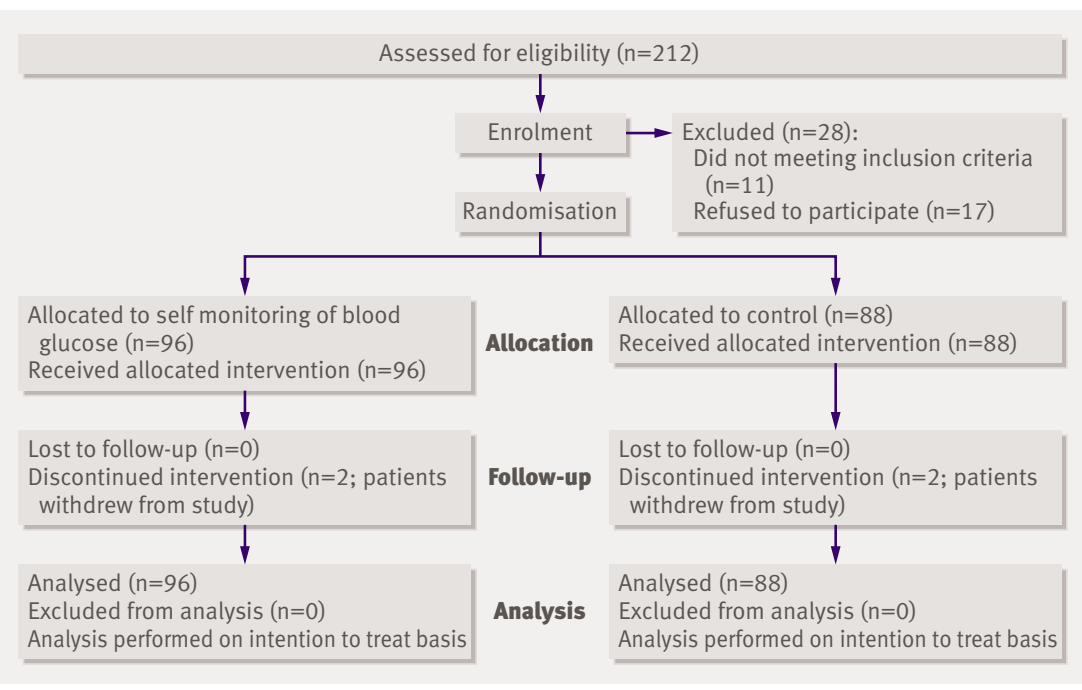

Fig 2 | Flow of patients through study nurse practitioners, dieticians, podiatrists, and medical staff. All patients were reviewed by the doctor, diabetes nurse practitioner, and dietician at three monthly intervals for one year. At each visit all aspects of diabetes care were reviewed including indices of glycaemic control $\left(\mathrm{HbA}_{1 \mathrm{c}}\right.$ for both groups and self monitoring results for the self monitoring group). Patients in the self monitoring group received ongoing advice and support in the appropriate interpretation of and response to their capillary glucose results.

We used an identical treatment algorithm for dietary and pharmacological management of glycaemia for both groups based on $\mathrm{HbA}_{1 \mathrm{c}}$ targets (figure 1). Blood concentrations of $\mathrm{HbA}_{1 \mathrm{c}}$, lipids, and electrolytes were measured at or before each clinic and results were discussed with patients in the context of the treatment targets. Measurement of $\mathrm{HbA}_{1 \mathrm{c}}$ was performed in the local hospital laboratory with a diabetes control and complications trial (DCCT) aligned $\mathrm{HbA}_{1 \mathrm{c}}$ assay. ${ }^{2}$ All laboratories participated in $\mathrm{HbA}_{1 \mathrm{c}}$ external quality assurance, which was satisfactory for the duration of the study. All other laboratory tests were also performed in the local hospital laboratory, where staff were blinded to treatment allocation.

At each three monthly visit patients completed a questionnaire survey incorporating the diabetes treatment satisfaction questionnaire, ${ }^{3}$ a modified version of the diabetes attitude scale, ${ }^{4}$ and the well-being questionnaire. ${ }^{5}$ The diabetes attitude scale included three of the seven subscales: seriousness of type 2 diabetes, blood glucose control and its implications, and impact of diabetes on patients' lives. ${ }^{4}$ The wellbeing questionnaire incorporated four subscales (depression, anxiety, energy, and positive wellbeing) and a total score of general wellbeing.

The study was powered to detect a $1 \%$ (1 unit) difference in $\mathrm{HbA}_{1 \mathrm{c}}(2 \mathrm{SD})$ between the groups at the 0.05 level (two tailed $t$ test) with a power in excess of 90\%. (A 50\% reduction in this standard deviation - that is, a narrowing of the $\mathrm{HbA}_{1 \mathrm{c}}$ distribution width as $\mathrm{HbA}_{1 \mathrm{c}}$ values converge on treatment targets-would detect a difference of $0.5 \%$ in $\mathrm{HbA}_{1 \mathrm{c}}$ with the above power.) We used Mplus univariate independent $t$ tests for statistical analysis and checked the results against non-latent growth models with time variant and time invariant covariates. ${ }^{6}$ We used three predetermined time invariant predictors: sex, age, monitoring status (that is, control or monitoring, with monitoring further broken down into two subgroups: patients who complied with the suggested monitoring regimen and patients who did not comply with the suggested monitoring regimen). Compliance was defined as a monitoring frequency of $>80 \%$ of that requested. In addition, we introduced the number of medications being taken for the control of $\mathrm{HbA}_{1 \mathrm{c}}$ as a time varying covariate. The analysis was performed on an intention to treat basis, with missing data imputed through the use of full information maximum likelihood. Psychological indices were examined by analysis of covariance with the measurement models held invariant across time. Differences between groups in use of oral 
Table 1|Baseline characteristics of patients with newly diagnosed diabetes according to self monitoring or no monitoring (control) of blood glucose. Figures are means (SD) unless stated otherwise

\begin{tabular}{lcc} 
& Monitoring group & Control \\
No of patients (men/women) & $96(55 / 41)$ & $88(56 / 32)$ \\
\hline Age (years) & $57.7(11.04)$ & $60.9(11.5)$ \\
\hline Body mass index & $34(6.98)$ & $32(6.23)$ \\
\hline$\% \mathrm{HbA}_{1 \mathrm{c}}$ & $8.8(2.1)$ & $8.6(2.3)$ \\
\hline
\end{tabular}

hypoglycaemic drugs were assessed by Pearson's $\chi^{2}$ test.

\section{RESULTS}

Of 212 consecutive participants approached between January 2003 and July 2005, 195 were recruited and $184(86.7 \%$ of those approached) proceeded to randomisation (96 to self monitoring) (fig 2). Four participants (two in each group) failed to complete the study.

There was no significant difference in baseline $\mathrm{HbA}_{1 \mathrm{c}}$, age, or sex between the groups, although participants in the self monitoring group had a higher baseline body mass index (BMI) (mean (SD) 34 (7) v 32 (6.2), table 1).

Of the 96 participants in the self monitoring group, 63 carried out more than $80 \%$ of the requested blood glucose monitoring (that is, four fasting and four postprandial readings a week).

\section{Outcome measures}

Although $\mathrm{HbA}_{1 \mathrm{c}}$ fell within each group, there were no significant differences between the groups at any time point, with mean (SD) values at 12 months of $6.9(0.8) \%$ $v 6.9(1.2) \%$ for the self monitoring versus control groups (table 2). The 95\% confidence interval for the difference at 12 months in $\mathrm{HbA}_{1 \mathrm{c}}$ between groups was $-0.25 \%$ to $0.38 \%$.

The measures of depression and anxiety were scored on a 100 point scale with the measure at the final time point regressed on to the baseline measure, monitoring status, and sex. All models provided an adequate description of the data. Participants in the self monitoring group were more depressed, scoring 6 points higher (that is, $6 \%$ ) on the depression subscale of the well-being questionnaire at 12 months $(\mathrm{P}=0.01)$, and there was a trend towards increased anxiety (table 3). There were no significant (0.05 level) differences between groups on any of the other subscales, in the mean scores on treatment satisfaction,

\begin{tabular}{|c|c|c|c|c|}
\hline Time (months) & Monitoring & Control & $P$ value & Mean difference $(95 \% \mathrm{Cl})$ \\
\hline 0 & $8.8(2.1)$ & $8.6(2.3)$ & 0.68 & $-0.33(-0.77$ to 0.51$)$ \\
\hline 3 & $7.2(1.1)$ & $7.1(1.2)$ & 0.50 & $0.18(-0.47$ to 0.23$)$ \\
\hline 6 & $7.0(0.9)$ & $7.0(1.1)$ & 0.82 & $0.04(-0.27$ to 0.35$)$ \\
\hline 9 & $6.9(0.8)$ & $7.1(1.4)$ & 0.30 & $0.19(-0.16$ to 0.54$)$ \\
\hline 12 & $6.9(0.8)$ & $6.9(1.2)$ & 0.69 & $0.07(-0.25$ to 0.38$)$ \\
\hline
\end{tabular}

or on any of the diabetes attitude subscales. There were no differences between groups in the incidence of reported hypoglycaemia at any time points or in use of oral hypoglycaemic drugs (tables 4 and 5). Although there was a difference in BMI between groups at randomisation, after correction for the baseline value there were no significant differences at subsequent time points (table 6).

\section{DISCUSSION}

Self monitoring of blood glucose concentrations might contribute in two ways to the management of type 2 diabetes: by improving glycaemic control or by improving the patients' experience of diabetes. In this cohort of patients with newly diagnosed type 2 diabetes we were unable to identify any significant effect of self monitoring over one year on $\mathrm{HbA}_{1 \mathrm{c}}$, BMI, use of oral hypoglycaemic drugs, or reported incidence of hypoglycaemia. Furthermore, monitoring was associated with a $6 \%$ higher score on the well-being depression subscale.

\section{Strengths of study}

We investigated the role of self monitoring in a cohort of patients with newly diagnosed type 2 diabetes, all of whom were therefore new to monitoring. We randomised a high proportion of eligible participants (86.7\%), suggesting that the cohort was representative of newly presenting patients in the community. The drop-out rate was low $(2.2 \%)$ and concordance with the monitoring regimen in the intervention group was high. We used a structured drug treatment algorithm based on $\mathrm{HbA}_{1 \mathrm{c}}$ targets for both groups. The core diabetes educational programme was identical to that administered under standard care, although education on monitoring was provided to those randomised to the monitoring group only. The starting $\mathrm{HbA}_{1 \mathrm{c}}$ was high $(8.8 \%$ and $8.6 \%$ in self monitoring and control groups, respectively) and both groups attained a satisfactory $\mathrm{HbA}_{1 \mathrm{c}}$ level of $6.9 \%$. We therefore think that the results of this study have general applicability.

\section{Comparison with other studies}

Previous studies on the efficacy of self monitoring have varied in design and have included non-randomised trials (both prospective and retrospective) and randomised controlled trials. ${ }^{7-19}$ The range of designs used in these studies reflects the difficulty of isolating the effect of a single home intervention in a condition in which

\begin{tabular}{|c|c|c|}
\hline Item & $\beta$ coefficient* (SE) & $P$ value \\
\hline Depression & $6.05(2.37)$ & 0.011 \\
\hline Anxiety & $5.86(3.19)$ & 0.07 \\
\hline Positive wellbeing & $4.16(2.88)$ & 0.15 \\
\hline Energy & $-0.84(2.83)$ & 0.77 \\
\hline
\end{tabular}

*All variables scored on 100 point scale and therefore $\beta$ coefficient corresponds to \% change associated with monitoring. 
Table 4 | Number of patients who reported hypoglycaemia (total number of hypoglycaemia episodes reported) according to self monitoring and no monitoring (control) of blood glucose

\begin{tabular}{lcc} 
Time (months) & Monitoring & Control \\
0 & $1(3)$ & $0(0)$ \\
\hline 3 & $5(10)$ & $2(8)$ \\
\hline 6 & $3(5)$ & $4(8)$ \\
\hline 9 & $5(9)$ & $1(6)$ \\
\hline 12 & $4(4)$ & $6(14)$ \\
\hline
\end{tabular}

patients' motivation, self management behaviour, and concordance with a prescribed drug regimen play a central role in effective treatment.

The retrospective ROSSO study found that self monitoring was associated with a reduction in both fatal and non-fatal (microvascular and macrovascular) events in 3268 patients over a mean review period of 6 . 5 years. ${ }^{19}$ The non-randomised retrospective study design, however, makes it difficult to exclude the possibility that more motivated patients opt to monitor and the fact of monitoring might therefore simply be a marker of generally beneficial self management behaviour. Randomised controlled trials offer a more robust tool for the investigation of self monitoring.

The small number of such trials undertaken, however, have varied in quality and provided conflicting results, though a meta analysis suggested a nonsignificant $0.39 \%$ (95\% confidence interval $0.21 \%$ to $0.56 \%$ ) reduction in $\mathrm{HbA}_{1 \mathrm{c}}$ in favour of monitoring, which would equate to $14 \%$ reduction in risk of microvascular complications. ${ }^{2021}$ There was clinical heterogeneity between the trials studied in both baseline characteristics and interventions. ${ }^{18}$ Educational interventions often differ between the self monitoring and control group, making it difficult to isolate the effect of self monitoring. ${ }^{1112}$ Two recent large studies (ASIA and DIGEM) have provided differing results on the role of self monitoring. ${ }^{11}{ }^{13}$ The ASIA study of 689 patients with established type 2 diabetes found significant reductions in $\mathrm{HbA}_{1 \mathrm{c}}$ from baseline in both self monitoring $(-0.88 \%)$ and control $(-0.6 \%)$ groups with a $0.3 \%$ reduction between groups in favour of monitoring at 24 weeks. ${ }^{11}$ The improvement in $\mathrm{HbA}_{1 \mathrm{c}}$ in the control group suggested that pre-existing management had been suboptimal and that management administered under the study protocol differed from usual care. Furthermore the ASIA study had a high drop-out rate $(48 \%$ in the self monitoring group, $40 \%$ in the control), which limits the general applicability of the findings. In contrast, the well designed DIGEM trial of 453 patients found no benefit of self monitoring (with or without structured education) in patients with established and well controlled type 2 diabetes, ${ }^{13}$ although the mean starting $\mathrm{HbA}_{1 \mathrm{c}}$ was low $(7.5 \%)$, which would have reduced the sensitivity for detecting an effect of monitoring.

An important difference between these randomised controlled trials and the present study is that our study included a rigorous treatment algorithm for the management of glycaemic control based on the target $\mathrm{HbA}_{1 \mathrm{c}}$. The success of this algorithm is shown by the reduction in mean $\mathrm{HbA}_{1 \mathrm{c}}$ in both groups to the satisfactory level of $6.9 \%$ at 12 months. The use of an effective and uniformly applied treatment regimen possibly minimises any potential benefit conferred by monitoring.

All studies to date, however, have included people with established diabetes, and it is unclear to what extent results could be extrapolated to newly presenting patients. ${ }^{22}$ Recruitment protocols in such studies generally excluded those who were already actively monitoring or who had recently monitored. This introduced a potential selection bias by excluding patients who had found monitoring beneficial, and by including patients who may have had previous experience of monitoring but rejected it as unhelpful..$^{7-12}$ The effect of any such bias would be to underestimate the benefit of self monitoring. We removed any such potential bias by recruiting only those patients with a new diagnosis of diabetesandwho had not previously performed self monitoring.

Anecdotal and other evidence suggests that some patients consider monitoring uncomfortable, intrusive, and unpleasant. ${ }^{2324}$ An interesting finding of our study was that monitoring was associated with a $6 \%$ higher score on a depression subscale and a trend towards increased anxiety, although satisfaction with treatment was unchanged. This supports the results of Franciosi et

Table 5 | Use of oral hypoglycaemic drugs in patients with newly diagnosed diabetes according to self monitoring or no monitoring (control) of blood glucose

\begin{tabular}{|c|c|c|c|}
\hline & Monitoring & Control & P value* \\
\hline \multicolumn{4}{|c|}{ At baseline $†$} \\
\hline No drugs & 86 & 78 & 0.67 \\
\hline 1 drug & 8 & 7 & 0.91 \\
\hline 2 drugs & 0 & 2 & 0.14 \\
\hline \multicolumn{4}{|c|}{ At 12 months } \\
\hline No drugs & 34 & 29 & 0.95 \\
\hline 1 drug & 44 & 40 & 0.62 \\
\hline 2 drugs & 11 & 6 & 0.36 \\
\hline
\end{tabular}

*Pearson's $x^{2}$ cross tabulation between monitoring and control by number of drugs.

†Data missing for two patients in monitoring group and one in control group.

‡Data missing for seven in monitoring group and 13 in control group. 
Table $6 \mid$ Body mass index (mean* (SD) and predicted overall mean) at 3, 6, 9, and 12 months adjusted for baseline value in self monitoring or no monitoring (control) groups

\begin{tabular}{|c|c|c|c|c|}
\hline Time (months) & Monitoring & Control & $P$ value & $\begin{array}{c}\text { Predicted overall mean } \\
\qquad(95 \% \mathrm{Cl} \dagger)\end{array}$ \\
\hline 0 & $34.0(7.0)$ & $32(6.2)$ & 0.04 & - \\
\hline 3 & $33.0(6.5)$ & $31.5(6.1)$ & 0.56 & 31.83 to 32.93 (32.38) \\
\hline 6 & $33(6.3)$ & $31.4(6.1)$ & 0.75 & 31.71 to $32.86(32.28)$ \\
\hline 9 & $33.1(6.3)$ & $31.7(6.1)$ & 0.49 & 31.99 to 33.19 (32.59) \\
\hline 12 & $33.1(6.4)$ & $31.8(6.0)$ & 0.32 & 32.09 to 33.29 (32.69) \\
\hline
\end{tabular}

*Raw score standard deviations.

†Confidence interval based on overall predicted mean score.

al, ${ }^{16}$ who also found higher levels of distress, depressive symptoms, and anxiety in patients who self monitored, and the qualitative findings of Peel et al. ${ }^{25}$ This possible negative effect of monitoring might be important and merits further investigation. Given that glycaemic control rapidly improved to satisfactory levels during the study, the negative effect might relate less to feelings of powerlessness in the face of high blood glucose readings than to the enforced discipline of regular monitoring without any tangible gain. This possibility should be considered when patients with a new diagnosis of diabetes are introduced to monitoring.

The value of self monitoring in patients with a new diagnosis is an important practical issue given that in UK clinical practice patients are often introduced to monitoring at an early stage after diagnosis. ${ }^{2223}$ Our results suggest it is not associated with any improvement in glycaemic control in such patients and might be associated with reduced wellbeing.

The ESMON Study Group comprises Vivien Coates, Margaret Copeland, Brendan Bunting (University of Uster); Maurice O'Kane, Sandra McConnell, Kenneth Moles, Sharon Patton (Altnagelvin Hospitals Health and Social Services Trust); Michael Ryan, Fergal Tracey, Mary Glass, Lesley Hamilton (Causeway Hospital Trust); Randal Hayes, Pooler Archbold, Sharon Martin, Margaret Devlin, Sonia Cambridge (Belfast City Hospital Trust); and Roy Harper, Moira Campbell, Lynne Thomas (Ulster Hospital and Community Trust). The executive committee comprises Vivien Coates, Brendan Bunting, Mary Glass, Sharon Martin, Roy Harper, Maurice O'Kane, and Margaret Copeland.

Contributors: MJO'K and VEC had the original idea for the study and wrote the protocol with BB in conjunction with members of the ESMON study group. BB was the study statistician. VEC and MC managed the study. MJOK, BB, MC, and VEC analysed and interpreted the study data. MJO'K wrote the first draft of the manuscript. All members of the study executive committee reviewed the final draft of the manuscript. VEC is guarantor. Funding: Northern Ireland research and development office. MC was employed as a research associate as part of the funding allocation. The blood glucose meters were supplied free of charge by Johnson and Johnson, Milpitas, CA.

\section{WHAT IS ALREADY KNOWN ON THIS TOPIC}

Self monitoring of blood glucose concentration in type 2 diabetes is widely advocated as an adjunct to achieving good glycaemic control

Randomised trials on self monitoring have given conflicting results, have been limited to patients with established diabetes, and have rarely considered quality of life

\section{WHAT THIS STUDY ADDS}

Self monitoring of blood glucose in patients with newly diagnosed type 2 diabetes did not result in improved glycaemic control but was associated with a $6 \%$ higher score on a depression index
Competing interests: None declared.

Ethical approval: University of Ulster ethics committee.

Provenance and peer review: Not commissioned; externally peer reviewed.

1 IDF Clinical Guidelines Taskforce. Global guidelines for type 2 diabetes: recommendations for standard, comprehensive and minimal care. Diabet Med 2006;23:579-93.

2 Diabetes Control and Complications Trial Research Group. The effect of intensive treatment of diabetes on the development and progression of long term complications of insulin dependent diabetes mellitus. N Engl J Med 1993;329:977-86.

3 Bradley C. Diabetes treatment satisfaction questionnaire [DTSQ]. In: Bradley C, ed. The handbook of psychology and diabetes; a guide to psychological measurement in diabetes research and practice. Chur, Switzerland: Harwood Academic Publishers, 1994:111-32.

4 Anderson RM, Donnelly MB, Dedrick RF. Measuring the attitudes towards diabetes and its treatment. Patient Educ Couns 1990;16:231-45.

5 Bradley C. The well-being questionnaire. In: Bradley C, ed. The handbook of psychology and diabetes; a guide to psychological measurement in diabetes research and practice. Chur, Switzerland: Harwood Academic Publishers, 1994:89-109.

6 Muthén LK, Muthén BO. Mplus user's guide. 4th ed. Los Angeles: Muthén \& Muthén, 2006.

7 Fontbonne A, Billault B, Acosta M, Percheron C, Varenne P, Besse A, et al. Is glucose self-monitoring beneficial in non-insulin treated patients? Results of a randomized comparative trial. Diabetes Metab 1989;15:255-60.

8 Allen BT, DeLong ER, FeussnerJR. Impact of glucose self-monitoring on non-insulin treated patients with type 2 diabetes mellitus. Diabetes Care 1990;13:1044-50.

9 Muchmore DB, Springer J, Miller M. Self-monitoring of blood glucose in overweight type 2 diabetic patients. Acta Diabetol 1994;31:215-9.

10 Schwedes U, Siebolds M, Mertes G. Meal-related structured selfmonitoring of blood glucose: effect on diabetes control in non-insulintreated type 2 diabetic patients. Diabetes Care 2002;25:1928-32.

11 Guerci B, Drouin P, Grange V, Bougneres P, Fontaine P, Passa P, et al. Self-monitoring of blood glucose significantly improves metabolic control in patients with type 2 diabetes mellitus: the auto-surveillance intervention active [ASIA] study. Diabetes Metab 2003;29:587-94.

12 Davidson MB, Castellanos M, Kain D, Duran P. The effect of selfmonitoring of blood glucose concentrations on glycated haemoglobin levels in diabetic patients not taking insulin. Am J Med 2005;118:422-5.

13 Farmer A, Wade A, Goyder E, Yudkin P, French D, Craven A, et al. Impact of self monitoring of blood glucose in the management of patients with non-insulin treated diabetes: open parallel group randomised trial. BMJ 2007;335:132-6.

14 Gallichan MJ. Self-monitoring by patients receiving oral hypoglycaemic agents: a survey and comparative trial. Practical Diabetes 1994;11:28-30

15 Miles P, Everett J, Murphy J, Kerr D. Comparison of blood or urine testing by patients with newly diagnosed type 2 diabetes: patient survey after randomised crossover trial. BMJ 1997;315:348-9.

16 Franciosi M, Pellegrini F, De Beradis G, Belfiglio M, Calviere D, Di Nardo B, et al. The impact of blood glucose self-monitoring on metabolic control and quality of life in type 2 diabetic patients: an urgent need for better educational strategies. Diabetes Care 2001;24:1870-7.

17 Karter AJ, Ackerson LM, Darbinian JA, D’Agostino RB Jr, Ferrara A, Liu J, et al. Self monitoring of blood glucose levels and glycemic control: the Northern California Kaiser Permanente diabetes registry. Am J Med 1996;100:157-63.

18 Soumerai SB, Mah C, Zhang F, Adams A, Barton M, Fajtova V, et al. Effects of health maintenance organization coverage of self- 
monitoring devices on diabetes self-care and glycaemic control. Arch Intern Med 2004;164:645-52.

19 Martin S, Schneider B, Heineman L, Lodwig V, Kurth H-J, Kolb H, et al. Self-monitoring of blood glucose in type 2 diabetes and long term outcome: an epidemiological cohort study. Diabetologia 2005;49:271-8.

20 Welschen LMC, Bloemendal E, Nijpels G, DekkerJM, Heine RJ, Stalman WAB, et al. Self-monitoring of blood glucose in patients with type 2 diabetes who are not using insulin. Diabetes Care 2005;28:1510-7.

21 Stratton IM, Adler Al, Neil HA, Matthews DR, Manley SE, Cull CA, et al. Association of glycaemia with macrovascular and microvascular complications of type 2 diabetes (UKPDS 35): prospective observational study. BMJ 2000;321:405-12.
22 Heller SR. Self monitoring of blood glucose in type 2 diabetes. BMJ 2007;335:105-6.

23 Gulliford M, Latinovic R. Variations in glucose self-monitoring during oral hypoglycaemic therapy in primary care. Diabet Med 2004;21:685-90.

24 Watkins KW, Connell CM, Fitzgerald JT, Klem L, Hickey T, Ingersoll-Dayton B. Effect of adults' self regulation of diabetes on quality of life issues. Diabetes Care 2000;23:1511-5.

25 Peel E, Douglas M, Lawton J. Self monitoring of blood glucose in type diabetes: longitudinal qualitative study of patients' perspectives. $B M$ J 2007;335:493.

Accepted: 21 March 2008 